\title{
水热法制备 $\mathrm{TiO}_{2}$ 纳米线薄膜的光生阴极保护性能
}

\author{
朱燕峰杜荣归* 李 静 漆海清林昌健 \\ (厦门大学化学化工学院化学系, 固体表面物理化学国家重点实验室, 福建厦门 361005)
}

\begin{abstract}
摘要: 应用水热法在钛箔表面制备 $\mathrm{TiO}_{2}$ 纳米线薄膜, 采用场发射扫描电子显微镜、X 射线衍射和紫外-可见分 光光度法对薄膜进行表征, 用电极电位和电化学阻抗谱考察 $\mathrm{TiO}_{2}$ 光生阴极保护性能. 结果表明: 薄膜由纵横交 错的锐钠矿型 $\mathrm{TiO}_{2}$ 纳米线组成, 纳米线的直径约 $10 \mathrm{~nm}$. 在 $150{ }^{\circ} \mathrm{C}$ 下反应 $6 \mathrm{~h}$ 生成的 $\mathrm{TiO}_{2}$ 纳米线薄膜在 0.3 $\mathrm{mol} \cdot \mathrm{L}^{-1} \mathrm{Na}_{2} \mathrm{SO}_{4}$ 溶液和 $0.3 \mathrm{~mol} \cdot \mathrm{L}^{-1} \mathrm{Na}_{2} \mathrm{SO}_{4}+0.5 \mathrm{~mol} \cdot \mathrm{L}^{-1} \mathrm{HCOOH}$ 混合溶液中对与 $\mathrm{TiO}_{2}$ 薄膜耦连的 403 不锈钢均 有良好的阴极保护效应. $\mathrm{TiO}_{2}$ 膜所在溶液中含有 $\mathrm{HCOOH}$ 时, 可使耦连的 403 不锈钢在 $0.5 \mathrm{~mol} \cdot \mathrm{L}^{-1} \mathrm{NaCl}$ 溶液中 电极电位负移约 $545 \mathrm{mV}$, 界面反应电阻显著变小, 表明电解质溶液加人 $\mathrm{HCOOH}$ 可以增强 $\mathrm{TiO}_{2}$ 纳米线薄膜对 403 不锈钢的光生阴极保护效应.
\end{abstract}

关键词: 水热法; $\mathrm{TiO}_{2}$ 纳米线薄膜; 光生阴极保护; 403 不锈钢

中图分类号: 0646

\section{Photogenerated Cathodic Protection Properties of a $\mathrm{TiO}_{2} \mathrm{Nanowire}$ Film Prepared by a Hydrothermal Method}

\author{
ZHU Yan-Feng DU Rong-Gui* LI Jing QI Hai-Qing LIN Chang-Jian \\ (State Key Laboratory for Physical Chemistry of Solid Surfaces, Department of Chemistry, College of Chemistry and Chemical \\ Engineering, Xiamen University, Xiamen 361005, Fujian Province, P. R. China)
}

\begin{abstract}
A} \mathrm{TiO}_{2}$ film was synthesized on the surface of a Ti substrate by a hydrothermal method, followed by acid treatment and calcination. The properties of the $\mathrm{TiO}_{2}$ film were characterized by scanning electron microscopy, $\mathrm{X}$-ray diffraction, and ultraviolet-visible spectrophotometry. The photogenerated cathodic protection properties of the $\mathrm{TiO}_{2}$ film were investigated by electrochemical techniques. The corrosion performance of 403 stainless steel coupled to a $\mathrm{TiO}_{2}$ film photoanode in different solutions was evaluated by photogenerated potential and electrochemical impedance spectroscopy. The results showed that the $\mathrm{TiO}_{2}$ film was composed of many randomly-oriented anatase nanowires of about $10 \mathrm{~nm}$ in diameter. The $\mathrm{TiO}_{2}$ nanowire film prepared by the hydrothermal reaction at $150{ }^{\circ} \mathrm{C}$ for $6 \mathrm{~h}$ was used for the photogenerated cathodic protection of 403 stainless steel. When we coupled the steel in a $0.5 \mathrm{~mol} \cdot \mathrm{L}^{-1} \mathrm{NaCl}$ solution to the $\mathrm{TiO}_{2}$ film photoanode in a mixed solution containing $0.3 \mathrm{~mol} \cdot \mathrm{L}^{-1} \mathrm{Na}_{2} \mathrm{SO}_{4}$ and $0.5 \mathrm{~mol} \cdot \mathrm{L}^{-1} \mathrm{HCOOH}$, its potential decreased by $545 \mathrm{mV}$. Additionally, the charge transfer resistance of the electrode reaction process for the coupled steel decreased considerably. The results also indicated that the $\mathrm{HCOOH}$ in the mixed solution improved the photogenerated cathodic protection of the $\mathrm{TiO}_{2}$ film photoanode.
\end{abstract}

Key Words : Hydrothermal method; $\mathrm{TiO}_{2}$ nanowire film; Photogenerated cathodic protection; 403 stainless steel

\footnotetext{
Received: April 16, 2010; Revised: May 28, 2010; Published on Web: July 13, 2010.

"Corresponding author. Email: rgdu@xmu.edu.cn; Tel: +86-592-2189192.

The project was supported by the National High Technology Research and Development Program of China (2009AA03Z327) and National Key Technology R\&D Program of China (2007BAB27B04).

国家高技术研究发展专项(2009AA03Z327), 国家科技支撑计划项目(2007BAB27B04)资助

(C) Editorial office of Acta Physico-Chimica Sinica
} 
纳米二氧化钛半导体以其优越的光电性能引起 了人们的广泛关注, 并成功应用于光催化降解有机 物 $^{[1-2]}$ 、光解水 ${ }^{[3-4]}$ 、太阳能电池 ${ }^{[5-6]}$ 、气敏传感器 ${ }^{[7-8]}$ 和生 物医用材料 ${ }^{[9-10]}$ 等领域. 例如, Wen 等 ${ }^{[11]}$ 采用溶胶凝 胶的方法制备了 I-F 共掺杂的 $\mathrm{TiO}_{2}$ 涂层, 应用于亚 甲基蓝(MB)的降解, 结果表明共掺杂的 $\mathrm{TiO}_{2}$ 膜比 纯 $\mathrm{TiO}_{2}$ 有更高的光催化活性. Uchida 等 ${ }^{[12]}$ 利用水热 法合成了 $\mathrm{TiO}_{2}$ 纳米管, 并表明制备的纳米管与未 处理前的粉末相比拥有更大的比表面积, 可以吸附 更多的染料, 提高光转换率. 李海龙等 ${ }^{[13}$ 研究了贵金 属 $\mathrm{Ag}$ 对 $\mathrm{TiO}_{2}$ 涂层性能的影响, 发现 $\mathrm{Ag}$ 修饰的 $\mathrm{TiO}_{2}$ 纳米管 $(\mathrm{Ag} / \mathrm{TNTs})$ 在可见光区域有较好的吸收, 光催化降解甲基橙表明 $\mathrm{Ag} / \mathrm{TNTs}$ 的光催化活性显 著高于纯的 TNTs. 近年来, 人们发现 $\mathrm{TiO}_{2}$ 涂层在金 属腐蚀保护方面也有光明的应用前景 ${ }^{[14-16]}$. 光照 时, $\mathrm{TiO}_{2}$ 价带电子就会被激发到导带, 在价带上产 生相应的空穴, 当 $\mathrm{TiO}_{2}$ 薄膜与某种金属相连时, 导 带的电子进人金属, 使金属的腐蚀电位降低, 从而抑 制或减少该金属的溶解. 将纳米 $\mathrm{TiO}_{2}$ 半导体材料的 光电特性应用于金属的保护, 相当于起到了一种阴 极保护效应, 也就是光生阴极保护作用. 与牺牲阳 极保护法不同的是 ${ }^{[17]}, \mathrm{TiO}_{2}$ 膜在产生阴极保护过程 中本身并不发生溶解, 有益于实现资源的可持续利 用. Ohko 等 ${ }^{[18]}$ 采用喷雾热解法在 304 不锈钢表面制 备 $\mathrm{TiO}_{2}$ 半导体薄膜, 发现紫外光照射时, 304 不锈 钢耐蚀性能提高; Zhou 等 ${ }^{[19]}$ 采用溶胶-凝胶法在 304 不锈钢表面构筑了 $\mathrm{TiO}_{2}-\mathrm{WO}_{3}$ 的纳米复合涂层, 提 高了不锈钢在 $\mathrm{NaCl}$ 溶液中的耐蚀性; $\mathrm{Li}$ 等 ${ }^{[20]}$ 应用阳 极氧化法在钛箔表面制备 $\mathrm{N}$ 掺杂的 $\mathrm{TiO}_{2}$ 纳米阵列 在太阳光照射下对 $316 \mathrm{~L}$ 不锈钢具有一定的阴极保 护作用.

虽然 $\mathrm{TiO}_{2}$ 薄膜光生阴极保护性能的发现和研 究已有多年, 但相关的报道仍较少. 存在的主要问题 是如何提高 $\mathrm{TiO}_{2}$ 薄膜光生阴极保护性能(重要指标 是被保护金属电极电位降低的数值). 为此, 人们一 般采用改进制备方法 ${ }^{[18-20]}$, 或对 $\mathrm{TiO}_{2}$ 薄膜进行改 性 ${ }^{[19-20]}$, 以增强其阴极保护效果. 目前, 制备 $\mathrm{TiO}_{2}$ 膜 层往往成本较高、或可能造成环境污染, 有时膜层易 出现缺陷. 本工作应用环境友好的水热法于钛箔表 面制备网状 $\mathrm{TiO}_{2}$ 纳米线薄膜, 并对薄膜形貌、结构、 光电性能进行表征. 通过电化学测试, 重点考察 $\mathrm{TiO}_{2}$ 薄膜光生阳极对耦连的 403 不锈钢电极的光 生阴极保护效应及其薄膜所处的电解质溶液的影响
作用.

\section{1 实验部分}

\section{$1.1 \mathrm{TiO}_{2}$ 纳米线薄膜制备}

制备 $\mathrm{TiO}_{2}$ 纳米线薄膜的基体材料为厚度 0.1 $\mathrm{mm}$, 纯度 $>99.9 \%$ 的钛管, 由厦门达真磁记录有限公 司提供; 用于测试 $\mathrm{TiO}_{2}$ 膜层光生阴极保护效应的金 属材料是厚度为 $2 \mathrm{~mm}$ 的 403 不锈钢 (即 $1 \mathrm{Cr} 13$ ) 板, 天津元浩腾飞公司供应; 所用试剂均为分析纯; 实 验用水为二次去离子水.

$\mathrm{TiO}_{2}$ 纳米膜制备方法. 钛筞于室温下经 $10 \%(w)$ $\mathrm{HNO}_{3}+1 \%(w) \mathrm{HF}$ 溶液刻蚀处理 $1 \mathrm{~min}$ 后, 冲洗, 再依 次用丙酮、无水乙醇、去离子水超声波清洗. 处理后 的钛筞放人容积为 $40 \mathrm{~mL}$ 的聚四氟乙烯反应釜中, 加人 $20 \mathrm{~mL} 10 \mathrm{~mol} \cdot \mathrm{L}^{-1} \mathrm{NaOH}$ 溶液, 于 $150{ }^{\circ} \mathrm{C}$ 下反应 一定时间, 再自然冷却至室温. 取出钛䇤试样, 去离 子水清洗, 然后在 $0.1 \mathrm{~mol} \cdot \mathrm{L}^{-1} \mathrm{HNO}_{3}$ 溶液中浸泡 $8 \mathrm{~h}$. 取出后用去离子水清洗, 最后于 $450{ }^{\circ} \mathrm{C}$ 下煅烧 $2 \mathrm{~h}$.

403 不锈钢电极的制备方法. 先把钢板加工成 2 $\mathrm{cm} \times 1 \mathrm{~cm}$ 的长方形试样, 其表面依次用 400\#-1500\# 的水磨砂纸逐级打磨, 再用 $1.0 \mu \mathrm{m}$ 的抛光粉抛光至 镜面, 依次在丙酮、无水乙醇、去离子水中超声波清 洗 $30 \mathrm{~min}$, 最后存放在干燥器中备用.

\section{$1.2 \mathrm{TiO}_{2}$ 纳米线薄膜的表征}

采用日本日立公司 Hitachi S-4800 场发射扫描 电子显微镜 $(\mathrm{SEM})$ 观察 $\mathrm{TiO}_{2}$ 纳米线薄膜的形貌和 测试其膜厚; 利用荷兰 Philips 公司的 panalytical $X^{\prime}$ pert 转靶 $X$ 射线粉末衍射仪 $(X R D)$ 测试膜层 $\mathrm{TiO}_{2}$ 的晶型; 应用美国 Varian 公司生产的 Cary5000 型 紫外-可见-近红外分光光度计(UV-Vis-NIR spectrophotometer)测试膜层的光吸收性能.

\section{$1.3 \mathrm{TiO}_{2}$ 纳米线薄膜光生阴极保护性能的测试}

$\mathrm{TiO}_{2}$ 薄膜光生阴极保护性能的测试采用光生 电解池和腐蚀测试电解池组成的双电解池联用系 统. 表面有 $\mathrm{TiO}_{2}$ 薄膜的 $\mathrm{Ti}$ 箔试样为光生阳极, 处于 光生电解池中, 其中的电解质为 $0.3 \mathrm{~mol} \cdot \mathrm{L}^{-1} \mathrm{Na}_{2} \mathrm{SO}_{4}$ 或 $0.3 \mathrm{~mol} \cdot \mathrm{L}^{-1} \mathrm{Na}_{2} \mathrm{SO}_{4}+0.5 \mathrm{~mol} \cdot \mathrm{L}^{-1} \mathrm{HCOOH}$ 溶液. 腐 蚀测试电解池为三电极体系, 工作电极为 403 不锈 钢, 辅助电极为 $\mathrm{Pt}$ 电极, 参比电极为饱和甘录电极 $(\mathrm{SCE})$, 以 $0.5 \mathrm{~mol} \cdot \mathrm{L}^{-1} \mathrm{NaCl}$ 为电解质溶液. $\mathrm{TiO}_{2}$ 膜 光生阳极与 403 不锈钢电极通过导线连接, 光生电 解池与腐蚀测试电解池通过盐桥连接. 测试 $\mathrm{TiO}_{2}$ 纳 米薄膜对 403 不锈钢的阴极保护效应时, 分为暗态 

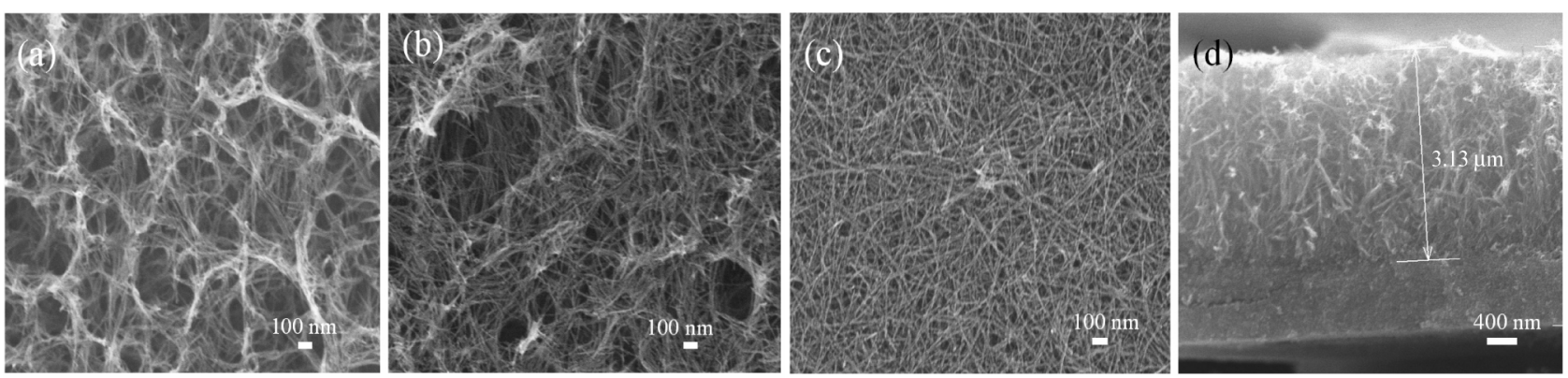

图 $1150{ }^{\circ} \mathrm{C}$ 水热反应不同时间制备的 $\mathrm{TiO}_{2}$ 纳米线薄膜的 $\mathrm{SEM}$ 图

Fig.1 SEM images of the $\mathrm{TiO}_{2}$ nanowire films prepared at $150{ }^{\circ} \mathrm{C}$ for different hydrothermal reaction time

(a) $2 \mathrm{~h}$, (b) $4 \mathrm{~h}$, (c) $6 \mathrm{~h}$; (d) cross-sectional view for the film prepared for $6 \mathrm{~h}$

与光照两种条件. 光照时以 $150 \mathrm{~W}$ 高压 $\mathrm{Xe}$ 灯作为 白光光源, 直接照射于光生电解池中 $\mathrm{TiO}_{2}$ 纳米薄膜 表面. 采用 Autolab PGSTAT30 电化学工作站测试 不锈钢的腐蚀电化学参数. 电化学阻抗(EIS)测试在 腐蚀电位或光生电位下进行, 激励信号为正弦, 扰动 电压 $10 \mathrm{mV}$, 频率范围为 $10^{5}-10^{-2} \mathrm{~Hz}$. 测试均在室温 下进行.

\section{2 结果与讨论}

\section{$2.1 \mathrm{TiO}_{2}$ 薄膜的表征}

图 1 为 $150{ }^{\circ} \mathrm{C} \mathrm{NaOH}$ 溶液中不同反应时间在 $\mathrm{Ti}$ 箔表面上制备的 $\mathrm{TiO}_{2}$ 薄膜的 SEM 图, 可以看出, 当反应时间为 $2 \mathrm{~h}$ 时, 膜表面呈现出由纳米线组成 的鸟巢状的结构, 线条没有一定的取向性, 处于纵横 交错(图 1(a)). 仔细观察, 可以发现这些纳米线的表 面并不是光滑的, 而是由一些颗粒组成, 纳米线形状 类似糖葫芦 ${ }^{[21]}$. 反应时间为 $4 \mathrm{~h}$ 时, $\mathrm{Ti}$ 表面膜鸟巢状 结构中的孔洞减小, 表面的纳米线增多, 其表面也较

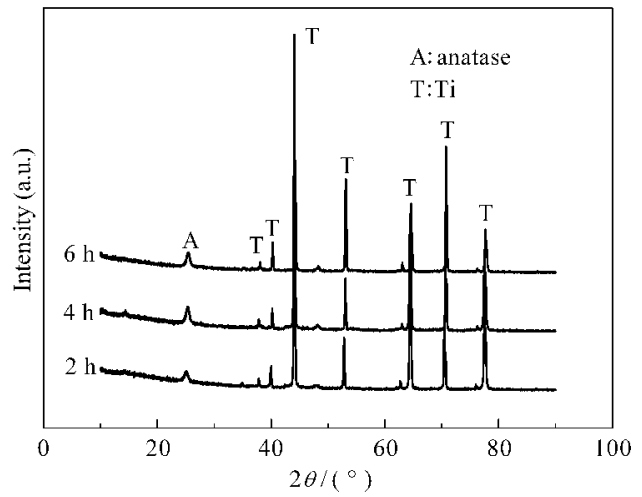

图 $2150{ }^{\circ} \mathrm{C}$ 水热反应不同时间制备的 $\mathrm{TiO}_{2}$ 纳米线 薄膜的 XRD 谱

Fig.2 XRD patterns of the $\mathrm{TiO}_{2}$ nanowire films prepared at $150{ }^{\circ} \mathrm{C}$ for different hydrothermal reaction time
光滑(图 l(b)), 表面膜也完全形成. 反应达到 $6 \mathrm{~h}$ 后, 钛箔表面形成了完整的纳米线薄膜(图 1(c)), 其中 纳米线直径约为 $10 \mathrm{~nm}$, 相应的侧面图显示膜层厚 度约 $3.13 \mu \mathrm{m}$ (图 1(d)). 因此, 水热法制备 $\mathrm{TiO}_{2}$ 纳米 线薄膜的反应时间应选择 $6 \mathrm{~h}$, 以获得稳定的结果.

图 2 为 $\mathrm{TiO}_{2}$ 纳米线薄膜的 XRD 谱图. 可以看 出, 图中存在着锐钛矿型 $\mathrm{TiO}_{2}$ 和纯钛的两种特征谱 峰, 表明纳米线主要成分是锐钛矿型 $\mathrm{TiO}_{2}$. 水热反 应时间为 $4 \mathrm{~h}$ 对应的 $\mathrm{TiO}_{2}$ 膜的特征峰位置与 $2 \mathrm{~h}$ 比 较并没有发生变化, 只是强度增大, 这是因为随着反 应时间的延长, 表面的纳米线逐渐增多, 这与 SEM 图显示的结果一致; 当反应时间增至 $6 \mathrm{~h}$ 时, $\mathrm{TiO}_{2}$ 膜 的特征峰同样不变, 而且与 $4 \mathrm{~h}$ 的谱峰比较, 其强度 也几乎不变. 这表明当反应时间达到 $4 \mathrm{~h}$ 后, 纳米线 薄膜已在 $\mathrm{Ti}$ 䇴表面完全形成, 正如上述 SEM 图所 显示的反应时间为 4 和 $6 \mathrm{~h}$ 时, $\mathrm{TiO}_{2}$ 膜的形貌基本 相同.

图 3 显示了不同反应时间制备的纳米线薄膜的

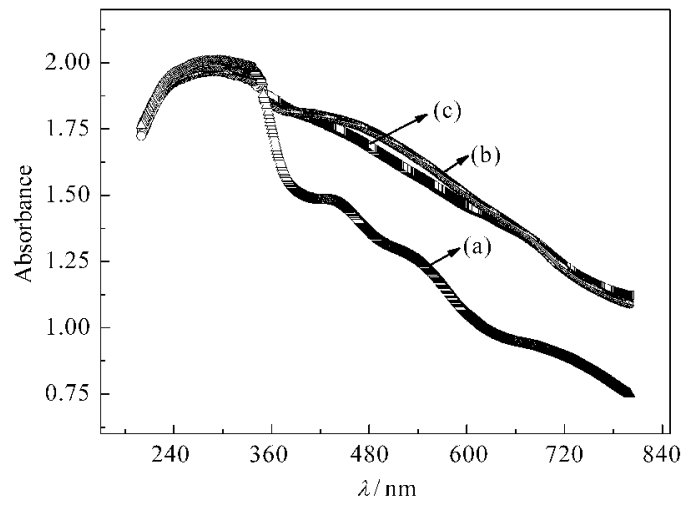

图 $3150{ }^{\circ} \mathrm{C}$ 水热反应不同时间制备的 $\mathrm{TiO}_{2}$ 纳米线薄膜的 紫外-可见吸收光谱

Fig.3 UV-Vis absorption spectra of the $\mathrm{TiO}_{2}$ nanowire films prepared at $150{ }^{\circ} \mathrm{C}$ for different hydrothermal reaction time

(a) $2 \mathrm{~h}$; (b) $4 \mathrm{~h}$; (c) $6 \mathrm{~h}$ 
紫外-可见吸收光谱. 可以看出, $\mathrm{TiO}_{2}$ 膜的主要吸收 波长为小于 $400 \mathrm{~nm}$ 的紫外光, 对应于锐钛矿型 $\mathrm{TiO}_{2}$ 的本征吸收. 水热反应 $4 \mathrm{~h}$ 时生成的 $\mathrm{TiO}_{2}$ 纳米 膜的吸收边与 $2 \mathrm{~h}$ 时比较有了显著的红移, 但仍然 主要吸收紫外光. 反应时间为 $6 \mathrm{~h}$ 制备的纳米膜的 吸收边与 $4 \mathrm{~h}$ 的情况几乎相同. 这是因为随着反应 时间的延长, 钛簿表面的 $\mathrm{TiO}_{2}$ 纳米线逐渐增多(如 图 1 所示), 有效表面积增大, 因此对人射光的吸收 就增强; 此外, 对于网状结构的纳米线薄膜, 光还可 以在其内部产生散射, 提高薄膜对光的吸收率 ${ }^{[21-22]}$, 由于薄膜对光的吸收增强, 其相应的光谱就表现为 吸收边红移.

\section{$2.2 \mathrm{TiO}_{2}$ 纳米线薄膜光生阴极保护性能}

\subsection{1 光生电位及其变化}

为考察 $\mathrm{TiO}_{2}$ 纳米线薄膜对金属的光生阴极保 护效应, 把表面有 $\mathrm{TiO}_{2}$ 纳米线薄膜 $\mathrm{Ti}$ 箔与 403 不 锈钢耦连后, 测量 403 不锈钢的电极电位 $E$, 也即测 试薄膜光生阳极在暗态和光照条件下对应耦连的不 锈钢的光生电位 $E_{\mathrm{ph}}$ 及其变化. 图 4 为 403 不锈钢 在 $0.5 \mathrm{~mol} \cdot \mathrm{L}^{-1} \mathrm{NaCl}$ 溶液中与水热反应时间为 $6 \mathrm{~h}$ 制得的处于不同介质中的 $\mathrm{TiO}_{2}$ 纳米线薄膜电极耦 连后电极电位随时间的变化曲线. 图中曲线(a)显示, 光生电解池中介质为 $0.3 \mathrm{~mol} \cdot \mathrm{L}^{-1} \mathrm{Na}_{2} \mathrm{SO}_{4}$ 溶液时, 用 白光照射 $\mathrm{TiO}_{2}$ 薄膜时, 不锈钢电极电位由原来的开 路电位(腐蚀电位) $15 \mathrm{mV}$ 迅速降低至约 $-370 \mathrm{mV}$, 随 后保持基本稳定, 这就表明 $\mathrm{TiO}_{2}$ 薄膜使 403 不锈钢 的电极电位显著降低, 起到了光生阴极保护作用. 产

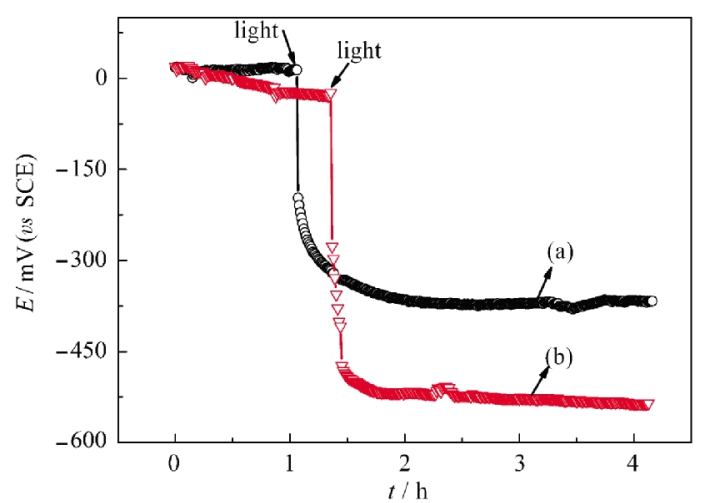

图 4403 不锈钢耦连电极在 $0.5 \mathrm{~mol} \cdot \mathrm{L}^{-1} \mathrm{NaCl}$ 溶液中 电极电位随时间变化

Fig.4 Time evolution of the potentials of 403 stainless steel in $0.5 \mathrm{~mol} \cdot \mathrm{L}^{-1} \mathrm{NaCl}$ coupled to $\mathrm{TiO}_{2}$ film electrode in different solutions (a) $0.3 \mathrm{~mol} \cdot \mathrm{L}^{-1} \mathrm{Na}_{2} \mathrm{SO}_{4}$; (b) $0.3 \mathrm{~mol} \cdot \mathrm{L}^{-1} \mathrm{Na}_{2} \mathrm{SO}_{4}+$ $0.5 \mathrm{~mol} \cdot \mathrm{L}^{-1} \mathrm{HCOOH}$
生这种现象的原因是由于光照射于 $\mathrm{TiO}_{2}$ 薄膜时, $\mathrm{TiO}_{2}$ 半导体价带的电子被激发到导带, 导带中的电 子通过导线传到 403 不锈钢的表面, 产生光生电子, 使不锈钢的电极电位降低 ${ }^{[23]}$. 当不锈钢表面电子数 目基本不变时, 光生电位就达到基本稳定, 不再随时 间变化. 如果 $\mathrm{TiO}_{2}$ 薄膜所处介质为 $0.3 \mathrm{~mol} \cdot \mathrm{L}^{-1}$ $\mathrm{Na}_{2} \mathrm{SO}_{4}+0.5 \mathrm{~mol} \cdot \mathrm{L}^{-1} \mathrm{HCOOH}$ 的混合溶液, 光照后, 403 不锈钢电极电位也迅速降低, 并且降低的数值 更大, 然后稳定在约 $-530 \mathrm{mV}$. 可见, 与 $0.3 \mathrm{~mol} \cdot \mathrm{L}^{-1}$ $\mathrm{Na}_{2} \mathrm{SO}_{4}$ 电解质溶液比较, 光生电解池的介质为混合 溶液时, 可使相应的 403 不锈钢产生的光生电位更 负, 表明此种情形 $\mathrm{TiO}_{2}$ 纳米线薄膜对不锈钢的光生 阴极保护效果更好. 产生这种效果的原因可解释为 电解质溶液中的甲酸不但作为空穴捕获剂降低了 $\mathrm{TiO}_{2}$ 纳米薄膜中电子和空穴的复合几率, 而且甲酸 还具有电流加倍效应以及在电极表面有较强的 吸附 ${ }^{[24}$. 这样, $\mathrm{TiO}_{2}$ 纳米薄膜就可以向耦连的不锈 钢提供更多的电子, 产生更负的光生电位, 达到更佳 的阴极保护作用.

\section{2 .2 电化学阻抗谱}

图 5 为 403 不锈钢在 $0.5 \mathrm{~mol} \cdot \mathrm{L}^{-1} \mathrm{NaCl}$ 溶液中 与受光照的 $\mathrm{TiO}_{2}$ 纳米薄膜耦连前后的电化学阻抗 谱. 从 Nyquist 谱图可看出, 其阻抗谱曲线只有一个 半圆弧, 根据此特征可采用图 6 的等效电路进行拟 合. 其中, $R_{\mathrm{s}}$ 代表溶液电阻; $R_{\mathrm{ct}}$ 代表不锈钢/溶液界 面电荷转移电阻, 其值大小反应了上述界面反应的 趋势; $Q_{\mathrm{dl}}$ 为空间电荷层电容. 从图中可以看出, 两种

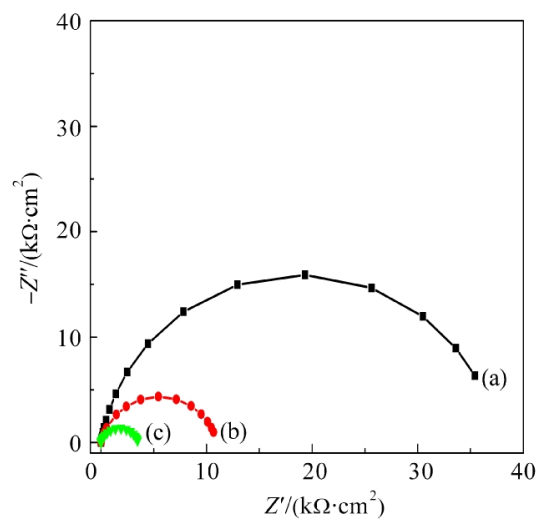

图 5403 不锈钢在 $0.5 \mathrm{~mol} \cdot \mathrm{L}^{-1} \mathrm{NaCl}$ 溶液中与 $\mathrm{TiO}_{2}$ 膜电 极耦连前后的 Nyquist 图

Fig.5 Nyquist plots of 403 stainless steel in $0.5 \mathrm{~mol} \cdot \mathrm{L}^{-1}$ $\mathrm{NaCl}$ solution under different conditions

(a) no coupling; (b) coupled to the $\mathrm{TiO}_{2}$ film in $0.3 \mathrm{~mol} \cdot \mathrm{L}^{-1} \mathrm{Na}_{2} \mathrm{SO}_{4}$ solution under illumination; (c) coupled to the $\mathrm{TiO}_{2}$ film in $0.3 \mathrm{~mol} \cdot \mathrm{L}^{-1}$ $\mathrm{Na}_{2} \mathrm{SO}_{4}+0.5 \mathrm{~mol} \cdot \mathrm{L}^{-1} \mathrm{HCOOH}$ under illumination 


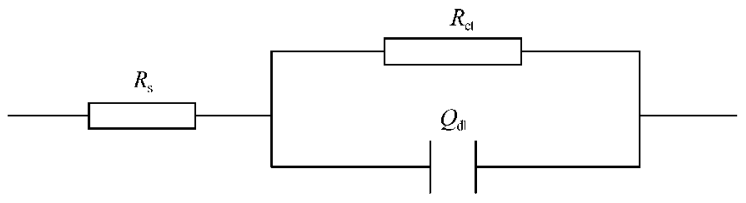

图 6403 不锈钢在 $0.5 \mathrm{~mol} \cdot \mathrm{L}^{-1} \mathrm{NaCl}$ 溶液中的电极过程 等效电路图

Fig.6 Equivalent circuit of 403 stainless steel in $0.5 \mathrm{~mol} \cdot \mathrm{L}^{-1} \mathrm{NaCl}$ solution

$R_{\mathrm{s}}$ : electrolyte solution resistance, $R_{\mathrm{ct}}$ : charge transfer resistance, $Q_{\mathrm{d}}$ : double-layer capacitance

情况下 Nyquist 图谱的形状基本上无变化, 但阻抗 值的大小差别显著. 根据等效电路对阻抗谱进行拟 合, 获得了 403 不锈钢电极耦连前的界面电荷转移 电阻 $R_{\mathrm{ct}}$ 为 $37.7 \mathrm{k} \Omega \cdot \mathrm{cm}^{2}$, 而与 $\mathrm{TiO}_{2}$ 膜光生阳极耦连 后光生电解池电解质分别为 $0.3 \mathrm{~mol} \cdot \mathrm{L}^{-1} \mathrm{Na}_{2} \mathrm{SO}_{4}$ 溶 液和 $0.3 \mathrm{~mol} \cdot \mathrm{L}^{-1} \mathrm{Na}_{2} \mathrm{SO}_{4}+0.5 \mathrm{~mol} \cdot \mathrm{L}^{-1} \mathrm{HCOOH}$ 混合 溶液时的 $R_{\mathrm{ct}}$ 分别为 $9.35 \mathrm{k} \Omega \cdot \mathrm{cm}^{2}$ 和 $3.53 \mathrm{k} \Omega \cdot \mathrm{cm}^{2}$. 可以看出, 403 不锈钢与 $\mathrm{TiO}_{2}$ 膜光生阳极相连后, $R_{\mathrm{ct}}$ 值减小了约 1 个数量级. 这是因为光照时, $\mathrm{TiO}_{2}$ 光生阳极产生的电子注人到 403 不锈钢的表面, 使 体系的总电流增大, 使不锈钢/溶液界面电荷转移速 度增大, 表现为电荷转移电阻降低, 阻抗值变小, Nyquist 图中圆弧半径也就变小, 而此时不锈钢表面 处于阴极保护状态, 其阳极溶解过程受到抑制. 当光 生电解池中电解质溶液中增加了 $\mathrm{HCOOH}$ 后, 如上 所述, 使电子在单位时间内从 $\mathrm{TiO}_{2}$ 膜转移到不锈钢 表面的数量增加, 相当于光生电解池和腐蚀测试电 解池之间的短路电流增加, 即电极反应速度更快, 因 此界面电荷转移电阻更小, 表现在 Nyquist 谱图的圆 弧半径也就更小. 因此, 介质为 $0.3 \mathrm{~mol} \cdot \mathrm{L}^{-1} \mathrm{Na}_{2} \mathrm{SO}_{4}+$ $0.5 \mathrm{~mol} \cdot \mathrm{L}^{-1} \mathrm{HCOOH}$ 混合溶液时, $\mathrm{TiO}_{2}$ 纳米薄膜对 金属具有更好的阴极保护效应.

\section{3 结 论}

应用水热法在钛箔表面构筑了网状锐钛矿型的 $\mathrm{TiO}_{2}$ 纳米线薄膜. 当水热反应时间达到 $6 \mathrm{~h}$ 后, $\mathrm{TiO}_{2}$ 膜完整覆盖了钛䇴表面, 其纳米线直径约 $10 \mathrm{~nm}$, 膜 厚约 $3.13 \mu \mathrm{m}$. 紫外-可见吸收光谱测试结果表明, 403 不锈钢在 $0.5 \mathrm{~mol} \cdot \mathrm{L}^{-1} \mathrm{NaCl}$ 溶液与作为光生阳 极的 $\mathrm{Ti}^{-} \mathrm{TiO}_{2}$ 纳米线薄膜耦连后, 在白光照射薄膜 条件下, 403 不锈钢电极电位显著负移, 界面电荷转 移电阻降低约 1 个数量级, 具有良好的阴极保护效 应. 特别是当 $\mathrm{Ti}_{-}-\mathrm{TiO}_{2}$ 纳米线薄膜所在的 $\mathrm{Na}_{2} \mathrm{SO}_{4}$ 溶 液中加人 $\mathrm{HCOOH}$ 后, 可以使耦连的不锈钢的电极
电位负移约 $545 \mathrm{mV}$, 比电解质为单一 $\mathrm{Na}_{2} \mathrm{SO}_{4}$ 溶液 时负移值多了 $160 \mathrm{mV}$, 表明介质中的甲酸对 $\mathrm{TiO}_{2}$ 纳米线薄膜的阴极保护效应具有显著的增强作用.

\section{References}

1 Srimala, S.; Roshanorlyza, H.; Zainovia, L. Thin Solid Films, 2009, 518(1-2): 16

2 Zhuang, H. F.; Lin, C. J.; Lai, Y. K.; Sun, L.; Li, J. Environ. Sci. Technol., 2007, 41(13): 4735

3 Chatterjee, D. Catal. Commun., 2010, 11(5): 336

4 Shaban, Y. A.; Khan, S. U. M. Int. J. Hydrogen. Energ., 2008, 33 (4): 1118

5 Lee, C. Y.; Hupp, J. T. Langmuir, 2010, 26(5): 3760

6 Wang, H. F.; Su, W. N.; Hwang, B. J. Electrochem. Commun., 2009, 11(8): 1647

7 Park, J. A.; Moon, J.; Lee, S. J. Kim, S. H.; Zyung, T.; Chu, H. Y. Mater. Lett., 2010, 64(3): 255

8 Seo, M. H.; Yussa, M.; Kida, T.; Huh, J. S. Shimanoe, K.; Yamazoe, N. Sensor. Actuat. B-Chem., 2009, 137(2): 513

9 Brammer, K. S.; Oh, S.; Cobb, C. J.; Bjursten, L. M.; van der Heyde, H.; Jin, S. Acta Biomater., 2009, 5(8): 3215

10 Peng, L.; Eltgroth, M. L.; LaTempa, T. J. Grimes, C. A.; Desai, T. A. Biomaterials, 2009, 30(7): 1268

11 Wen, C.; Zhu, Y. J.; Kanbara, T.; Zhu, H. Z.; Xiao, C. F. Desalination, 2009, 249(2): 621

12 Uchida, S.; Chiba, R.; Tomiba, M. Electrochemistry, 2002, 70(6): 418

13 Li, H. L.; Luo, W. L.; Chen, T. Acta Phys. -Chim. Sin., 2008, 24 (8): 1383 [李海龙, 罗武林, 陈 涛. 物理化学学报, 2008, 24 (8): 1383]

14 Tatsuma, T.; Saitoh, S. Ohko, Y.; Fujishima, A. Chem. Mater., 2001, 13(9): 2838

15 Shen, G. X.; Chen, Y. C.; Lin, C. J. Thin Solid Films, 2005, 489 $(1-2): 130$

16 Shen, G. X.; Chen, Y. C.; Lin, L.; Lin, C. J. Electrochim. Acta, 2005, 50(25-26): 5083

17 Park, H.; Kim, K. Y.; Choi, W. Chem. Commun., 2001, (3): 281

18 Ohko, Y.; Saitoh, S.; Tatsuma, T.; Fujishima, A. J. Electrochen. Soc., 2001, 148(1): B24

19 Zhou, M. J.; Zeng, Z. O.; Zhong, L. Corrosion Sci., 2009, 51(6): 1386

20 Li, J.; Yun, H.; Lin, C. J. J. Electrochem. Soc., 2007, 154(11): C631

21 Dong, X.; Tao, J.; Li, Y. Y.; Wang, T.; Zhu, H. Acta Phys. -Chim. Sin., 2009, 25(9): 1874 [董 祥, 陶 杰, 李莹漟, 汪 涛, 朱 宏. 物理化学学报, 2009, 25(9): 1874]

22 Fujita, K.; Konishi, J.; Nakanishi, K. Sci. Technol. Adv. Mater., 2006, 7(6): 511

23 Park, H.; Kim, K. Y.; Choi, W. J. Phys. Chem. B, 2002, 106(18): 4775

24 Leng, W. H.; Liu, D. P.; Cheng, X. F.; Zhu, W. C.; Zhang, J. Q.; Cao, C. N. Acta Metall. Sin., 2007, 43(7): 764 [冷文华, 刘东坡, 程小芳, 朱文彩, 张鉴清, 曹楚南. 金属学报, 2007, 43(7): 764] 